\title{
AN INTEGRAL FORMULA ON THE SCALAR CURVATURE OF ALGEBRAIC MANIFOLDS
}

\author{
JIH-HSIN CHENG
}

\begin{abstract}
It is proved in this note that if the scalar curvature of an $n$-dimensional algebraic complex submanifold is bigger than $n^{2}$, then it is totally geodesic.
\end{abstract}

0. Introduction. Let $\rho$ denote the scalar curvature of an $n$-dimensional compact connected Kaehlerian submanifold $M$ in a complex projective space with the standard Fubini-Study metric of constant holomorphic sectional curvature 1, denoted by $C P^{n+p}(1)$ where $p$ is the codimension. And, let $Q^{n}$ denote the hyperquadric which is defined by

$$
\left\{z=\left[z_{0}, z_{1}, \ldots, z_{n+1}\right] \in C P^{n+1}(1) \mid z_{0}^{2}+z_{1}^{2}+\cdots+z_{n+1}^{2}=0\right\}
$$

with the induced metric. In this note, we shall prove the following result.

THEOREM. With the above notations, we have

$$
\int_{M}\left[\rho-n^{2}\right][\rho-n(n+1)] * 1>0
$$

where * denotes the Hodge star operator and the equality holds if and only if $M$ is holomorphically isometric to either $C P^{n}(1)$ or $Q^{n}$.

Let $\|\sigma\|$ denote the length of the second fundamental form $\sigma$ of $M$ embedded in $C P^{n+P}(1)$, then we have the following corollary.

Corollary 1. $\int_{M}\|\sigma\|^{2}\left(\|\sigma\|^{2}-n\right) * 1>0$ and the equality holds if and only if $\|\sigma\|^{2}$ is identically equal to either 0 or $n$ and $M$ is holomorphically isometric to either $C P^{n}(1)$ or $Q^{n}$ respectively.

Proof. Observe that $\rho=n(n+1)-\|\sigma\|^{2}$ (cf. [4]).

Corollary 2. If $\rho>n^{2}$, then $M$ is totally geodesic.

Proof. Note that $\rho$ is not bigger than $n(n+1)$. Also see [4].

REMARK. In [4], Corollary 2 was conjectured by $K$. Ogiue even in the case where $M$ is complete.

1. Preliminaries. Let $M$ be a Kaehler manifold of complex dimension $n$ and $\left(\theta^{1}, \ldots, \theta^{n}\right)$ form a local field of unitary coframes, then the Kaehler metric $g$ and

Received by the editors March 6, 1980.

1980 Mathematics Subject Classification. Primary 53C55; Secondary 53C40. 
the associated Kaehler form $\phi$ are given respectively by

$$
\begin{gathered}
g=\frac{1}{2} \sum\left(\theta^{i} \otimes \bar{\theta}^{i}+\bar{\theta}^{i} \otimes \theta^{i}\right), \\
\phi=\frac{\sqrt{-1}}{2} \sum \theta^{i} \wedge \bar{\theta}^{i} .
\end{gathered}
$$

Let $\Theta_{j}^{i}=\sum R_{j k l}^{i} \theta^{k} \wedge \bar{\theta}^{l}$ be the curvature form of $M$. The Ricci tensor $S$ and the scalar curvature $\rho$ are given respectively by

$$
S=\frac{1}{2} \sum\left(R_{i j} \theta^{i} \otimes \theta^{\bar{j}}+\overline{R_{i j}} \overline{\theta^{i}} \otimes \theta^{j}\right) \text { and } \rho=2 \sum R_{i \bar{i}}
$$

where $R_{i j}=2 \sum R_{i k \bar{j}}^{k}$. Put $\gamma=(\sqrt{-1} / 4 \pi) \sum R_{i j} \theta^{i} \wedge \theta^{\bar{j}}$. Then its cohomology class $[\gamma]$ is the first Chern class $c_{1}$ (or $c_{1}(M)$ ) of $M$.

We define the $k$ th scalar curvature $\rho_{k}$ by $\rho_{k}=\left((2 \pi)^{k} / n !\right) *\left(\phi^{n-k} \wedge \gamma^{k}\right)$. It is easy to check $\rho_{0}=1, \rho_{1}=(1 / 2 n) \rho, \rho_{2}=(1 / 4 n(n-1))\left(\rho^{2}-2\|S\|^{2}\right)$ where $\|S\|$ denotes the length of the Ricci tensor $S$.

We need the following facts.

FACT 1 (B. Y. CHEN [1]). Let $M$ be an $n$-dimensional compact Kaehler manifold. Then

$$
\int_{M} \rho_{k} * 1=\frac{(4 \pi)^{n}}{n ! 2^{k}}\left(c_{1}^{k} \Omega^{n-k}\right)[M]
$$

where $\Omega$ denotes the normalized Kaehler class $(1 / 4 \pi)[\phi]$.

FACT 2 (B. Y. Chen AND K. OGIUE [2]). Let $M$ be an $n$-dimensional Kaehler manifold. Then $2 n\|S\|^{2} \geqslant \rho^{2}$ and the equality holds if and only if $M$ is Einsteinian.

Now, we also need the following basic result in algebraic geometry.

FACT 3 (A. J. Sommese [6] OR A. VAN DE VEN [7]). Let $L$ be a very ample line bundle on an algebraic surface $X$, and let $K_{X}$ be the canonical bundle of $X$. Then $K_{X} \otimes L$ is spanned by its sections if and only if $L$ is not one of the following:

(i) $O(1)$ or $O(2)$ on $C P^{2}$ where $O(1)$ denotes the hyperplane section bundle and $O(2)=O(1) \otimes O(1)$

(ii) a line bundle on a ruled surface, whose restriction to any fibre $C P^{1}$ is $O(1)$.

2. Proof of the Theorem. Straightforward computations give

$$
\left[\rho-n^{2}\right][\rho-n(n+1)]=\rho^{2}-\left(2 n^{2}+n\right) \rho+n^{3}(n+1)
$$

and

$$
\int_{M} \rho * 1=\int_{M} 2 n \rho_{1} * 1=\frac{(4 \pi)^{n}}{(n-1) !}\left(c_{1} \Omega^{n-1}\right)[M]
$$

Since $\rho_{2}=(1 / 4 n(n-1))\left(\rho^{2}-2\|S\|^{2}\right)$, by combining Facts 1 and 2 , we get the following estimation of the integral of $\rho^{2}$

$$
\int_{M} \rho^{2} * 1>\frac{(4 \pi)^{n} n}{(n-1) !}\left(c_{1}^{2} \Omega^{n-2}\right)[M]
$$


and equality holds if and only if $M$ is Einsteinian. Now putting (A), (B), and (C) together, we have

$$
\begin{aligned}
\int_{M}[\rho- & \left.n^{2}\right][\rho-n(n+1)] * 1 \\
& \geqslant \frac{(4 \pi)^{n} \cdot n}{(n-1) !}\left(\left[c_{1}-n \Omega\right]\left[c_{1}-(n+1) \Omega\right] \Omega^{n-2}\right)[M] .
\end{aligned}
$$

We shall show that the right-hand side of (D) is nonnegative after a lemma in algebraic geometry.

Let $L$ be the hyperplane section bundle over $M$, i.e. the pullback of $O_{C P^{n+p}}(1)$. Then $L$ is very ample.

LeMma. $K_{M} \otimes L^{n}$ is spanned unless $M$ is a linear subspace $C P^{n}$ of $C P^{n+p}$.

Proof. Suppose $K_{M} \otimes L^{n}$ is not spanned. Observe $\left.\left(K_{M} \otimes L\right)\right|_{M \cap H}=K_{M \cap H}$ by the adjunction formula, where $H$ is a generic hyperplane of $C P^{n+p}$. Then we see that $\left.\left(K_{M} \otimes L^{n}\right)\right|_{X}=K_{X} \otimes L_{X}^{2}$ is not spanned either for some $X=M \cap H_{1}$ $\cap \cdots \cap H_{n-2}$ where $H_{i}$ 's are generic hyperplanes of $C P^{n+p}, 1<i<n-2$, and $L_{X}$ denotes the restriction of $L$ to $X$. Now by Fact 3 , there are two cases to be discussed. Case (i). $X$ is biholomorphic to $C P^{2}$ and $L_{X}^{2}$ is either $O(1)$ or $O(2)$.

Clearly $L_{X}^{2}$ cannot be $O(1)$ since every line bundle over $C P^{m}$ has to be $O(l)$ for some integer $l$. Thus $L_{X}^{2}=O(2)$ and $L_{X}=O(1)$ which implies that the degree of $M$ in $C P^{n+p}$ is equal to 1 and $M$ has to be a linear subspace $C P^{n}$ of $C P^{n+p}$. The case (ii) in Fact 3 is impossible because $\left.L_{X}^{2}\right|_{C P^{1}}$ cannot be $O_{C P^{1}}(1)$ for the same reason as above. So we have shown our lemma.

Now, if $M$ is a linear $C P^{n}$, then the right-hand side of (D) vanishes since $c_{1}\left(C P^{n}\right)=(n+1) \Omega$. And that $K_{M} \otimes L^{n}$ is spanned tells us $K_{M} \otimes L^{n+1}$ is very ample since $L$ is very ample. Hence

$$
\begin{aligned}
c_{1}\left(K_{M} \otimes L^{n}\right) c_{1}\left(K_{M} \otimes L^{n+1}\right) & =\left[-c_{1}(M)+n c_{1}(L)\right]\left[-c_{1}(M)+(n+1) c_{1}(L)\right] \\
& =\left[c_{1}(M)-n \Omega\right]\left[c_{1}(M)-(n+1) \Omega\right]
\end{aligned}
$$

is nonnegative which implies the right-hand side of $(D)$ is also nonnegative. So we have proved the inequality in the theorem.

Now, if the equality in our integral formula holds, then

$$
0=\left(\left[n \Omega-c_{1}\right]\left[(n+1) \Omega c_{1}\right] \Omega^{n-2}\right)[M]
$$

which forces $n \Omega=c_{1}$ unless $M$ is a linear $C P^{n}$ by using the following fact: if $\phi$ is a nonnegative $(1,1)$ form and $\psi$ is a positive $(n-1, n-1)$ form, then $\int_{M} \phi \wedge \psi=0$ if and only if $\phi=0$. On the other hand, $M$ is Einsteinian since the equality of (c) holds. Now either $M$ is a linear $C P^{n}$ i.e. $M$ is holomorphically isometric to $C P^{n}(1)$ or $c_{1}=n \Omega$ which implies $M$ is biholomorphic to $Q^{n}$ as a hypersurface of a linear subspace $C P^{n+1}(1)$ of $C P^{n+p}(1)$ (S. Kobayashi and T. Ochiai [3]). And since $M$ is Einsteinian, $M$ has to be also isometric to $Q^{n}$ (B. Smyth [5]). Conversely, either $C P^{n}(1)$ or $Q^{n}$ surely makes the equality in our integral formula hold. So we complete the proof. 
Acknowledgement. The author would like to thank Professor A. J. Sommese for his valuable information on algebraic geometry.

APPENDIX. For $n=1$, we estimate the integral of $\rho^{2}$ by the Schwarz inequality.

$$
\left(\int_{M} \rho^{2} * 1\right)\left(\int_{M} * 1\right) \geqslant\left(\int_{M} \rho * 1\right)^{2} \text {. }
$$

Let $A=\int_{M} \rho * 1$ and $v=\int_{M} * 1$. Then $A=4 \pi c_{1}[M]=4 \pi(2-2 g)<8 \pi$ where $g$ is the genus of $M$, and $v=4 \pi d$ where $d$ is the degree of $M$. Hence

$$
\int_{M}(\rho-1)(\rho-2) * 1 \geqslant v\left(\frac{A}{v}-2\right)\left(\frac{A}{v}-1\right) \geqslant 4 \pi d\left(\frac{2}{d}-2\right)\left(\frac{2}{d}-1\right)>0
$$

and the equality holds if and only if $\rho$ is constant and equal to either 1 or 2 , which implies that $M$ is holomorphically isometric to either $Q^{1}$ or $C P^{1}(1)$ respectively.

\section{REFERENCES}

1. B. Y. Chen, Characterizations of Einstein Kaehler manifolds and applications, Atti Accad. Naz. Lincei Rend. Cl. Sci. Fis. Mat. Natur. 61 (1976), 592-595.

2. B. Y. Chen and K. Ogiue, Some characterizations of complex space forms in terms of Chern classes, Quart. J. Math. Oxford Ser. (2) 26 (1975), 459-464.

3. S. Kobayashi and T. Ochiai, Characterizations of complex projective space and hyperquadratics, J. Math. Kyoto Univ. 13 (1973), 31-47.

4. K. Ogiue, Differential geometry of Kaehler submanifolds, Adv. in Math. 13 (1974), $73-114$.

5. B. Smyth, Differential geometry of complex hypersurfaces, Ann. of Math. 85 (1967), 246-266.

6. A. J. Sommese, Hyperplane sections of projective surfaces. I, The adjunction mapping. Duke Math. J. 46 (1979), 377-401.

7. A. Van de Ven, On the 2-connectedness of very ample divisors on a surface, Duke Math. J. 46 (1979), 403-407.

Department of Mathematics, University of Notre Dame, Notre Dame, Indiana 46556 